\title{
XLV. On the deflexion by magnetic force of the electric discharge through gases
}

\author{
J. Henry M.A. B.E. (R.U.I.)
}

To cite this article: J. Henry M.A. B.E. (R.U.I.) (1898) XLV. On the deflexion by magnetic force of the electric discharge through gases, Philosophical Magazine Series 5, 46:282, 429-452, DOI: $10.1080 / 14786449808621216$

To link to this article: http://dx.doi.org/10.1080/14786449808621216

曲 Published online: 08 May 2009.

Submit your article to this journal $[\pi$

Џ Article views: 3

Q View related articles $\square$ 


\title{
PHILOSOPHICAI, MAGAZINE
}

\author{
AND \\ JOURNAL OF SCIENCE.
}

[FIFTH SERIES.]

NOVEMBER 1898.

XLV. On the Deflexion by Magnetic Force of the Electric Discharge through Gases. By J. Henny, M.A., B.E. (R.L.I.), Trinity College, Cambridge; 1851 Exhibition Science Scholar, Queen's College, Galway*.

THE experiments described in the following paper were undertaken for the purpose of finding the explanation of the change produced in the magnetic deflexion of the electric discharge between a pair of electrodes in a rarefied gas, when the gas carrying the discharge is changed: this effect of the gas on the magnetic deflexion of the discharge through it had been noticed by Professor Thomson.

It is well known that the electric discharge between a pair of electrodes in a rarefied gas is deflected by a magnetic force at right angles to the direction of discharge, in somewhat the same way as a flexible and elastic conducting string would be, if it were stretched between the electrodes and carried a current in the same direction as that in which the discharge passes through the gas; the discharge in the gas, like the string, is deflected from the straight line between the electrodes while the magnetic force acts, and retarns to the straight path again as soon as the magnetic force is removed ('Recent Researches,' p. 131, par. 127).

\section{Description of Apparatus.}

The apparatus used in my experiments consisted of :A vacuum-tube with an air-pump and a mercury-manometer

* Communicated by Prof. J. J. Thomson, F.R.S.

Phil. Mag. S. 5. Vol, 46. No. 282. Nov, $1898 . \quad 2 \mathrm{H}$ 
tube attached; a pair of coils for producing a magnetic field; an induction-coil for producing the discharge, a galvanometer for measuring it, and an electrostatic voltmeter for giving the difference of potential between the electrodes of the discharge ; and a camera for photographing the discharge.

The vacunm-tube is shown in fig. 1 . It was found that in a narrow tube the direction of the discharge for high pressures of the gas was very unsteady, owing to the walls of the tube becoming electrified; so for this reason, and also to allow room for the discharge to be deflected without coming too close to the glass, a wide tube had to be used. An ordinary bell-jar, fitted as shown in fig. 1, made a very convenient

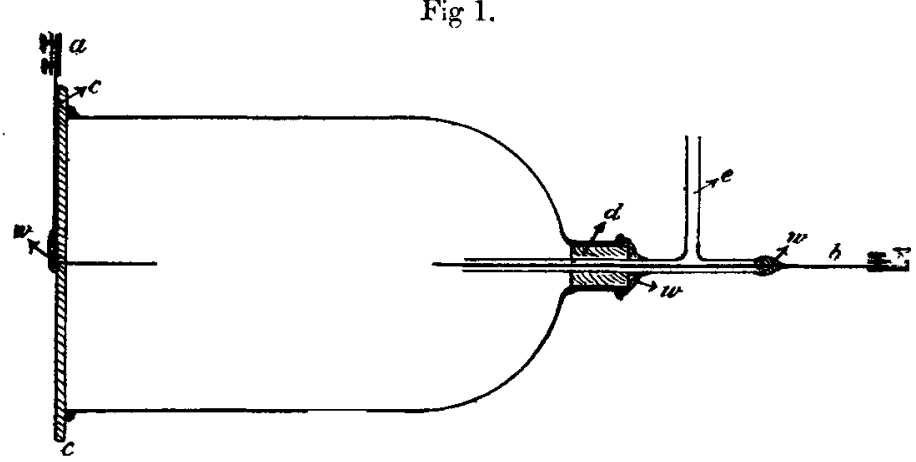

$a$ and $b$ are the terminals : $c, c$ the ebonite plate; $d$ the ebonite plug; $e$ the tube leading to the air-pump; $v$ sealing-wax. Diameter of bell-jar $9 \cdot 4$ cms.

form. The ebonite plug, $d$, through which the three-way glass tube passes, was made air-tight in the neck of the jar with sealing-wax ; the glass tube contained the electrode, $b$, and also allowed of gas being admitted to, or pumped out of, the bell-jar by the side tube, e. The electrode was fixed in the tube with the sealing-wax, $w$; but by softening the wax it could be easily shifted in or out, so as to change the length of the discharge.

The bottom of the jar was closed by pressing a plate of ebonite (c) turned plave, against the ground glass rim of the jar. The joint was at first made air-tight with sealing-wax, but grease was tound to answer the purpose quite as well, and $i t$ was much easier to work with.

The electrode, $u$, passed through the centre of the ebonite plate, and was secured with sealing-wax. The tube $e$ communicated with a mercury manometer, the air-pump, and the gas-supplying apparatus, through an arrangement of three-way tubes with stopcocks. 
The exhaustion in most of the experiments was made with an oil air-pump capable of producing a vacuum of less than $1 \mathrm{~mm}$. of mercury.

Two large coils of thick copper wire, capable of carrying a current of ten or twelve amperes for a short time, were used for producing the magnetic force to deflect the discharge. These coils were wound on wooden bobbins with 150 turns in each coil, and with a mean radius of $17 \mathrm{cms}$. each. They were fixed in a frame with their planes parallel, and perpendicular to the line joining their centres, at a distance apart of $17 \mathrm{cms}$, the mean radius of either coil,-Helmboltz's arrangement for producing a uniform field about the middle point of the axis of the coils.

The current in the coils was given by an ammeter in series with them.

The discharge was produced by a Ruhmkorff's coil, and the mean current in the discharge was measured by a galvanometer in the discharge-circuit.

In the experiments the discharge-tube was placed so that the middle point of the discharge was as near the middle point of the axis of the coils as possible, and with the direction of the discharge at right angles to that axis.

\section{Preliminary Lixperiments.}

Some prelimmary experiments were made with different gases in the discharge-tube, and for a given magnetic force, the deflexion of the discharge produced in each gas was observed. As the deflexions could not be very accurately compared with the eye alone, a camera was employed; it was permanently fixed and focused on the discharge so that a photograph could be obtained at any time by simply uncapping the lens. In order to compare the deflexions produced by a constant magnetic force in the different gases, the mean current in the discharge, as measured by the galvanometer, was kept constant by varying the pressure of the gas in the discharge-tube.

The following table gives the gases used, arranged in order of the magnitude of the deflexions obtained in them by the method stated above: the deflexion in hydrogen was least.

(1) $\mathrm{H}$.

(2) Cl?
(4) NO.

(5) $\left\{\begin{array}{l}\mathrm{Br} \\ \mathrm{Co}\end{array}\right.$
(6) $\mathrm{NH}_{2}$.

(7) $\left\{\begin{array}{l}\mathrm{CO}_{2} \\ \mathrm{SO}_{2}\end{array}\right.$ ?

The position of the gases marked thus ? in the above table is rather doubtful, as the oil-pump could not be used for $2 \mathrm{H} 2$ 
exhausting the discharge-tube of these gases owing to their deleterious effect on the pump.

Figs. 2, 3, and 4 show the deflexions obtained in hydrogen, air, and carbonic acid gas respectively; the defexion in chlorine was much nearer that in air than in hydrogen.

Fig. 2.

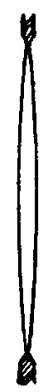

Fig. 3.

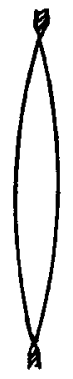

Fig. 4.

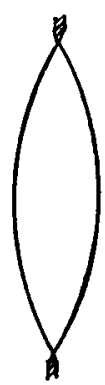

These figures show the discharge deflected to both sides of the straight path, as in the photos. Each plate was exposed to the discharge deflected first to one side, then to the other, by reversing the current in the magnetic-field coils. The figures are the same size as the photos.

The actual distance between the terminals was Fig. 5 . $8.8 \mathrm{cms}$.

During these experiments I noticed that the curvature of the deflected discharge was not uniform but was always greater at the upper electrode, as shown in fig. 5 , the discharge being vertical. It did not matter whether $b$ was the positive or the negative electrode.

The distortion was always much greater for discharges passing through gases at high pressures than at low pressures.

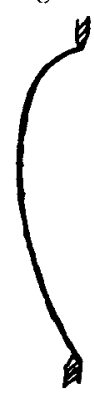

Bending of the Discharge by Convection-Currents.

To try if the distortion, above referred to, was caused by convection-currents due to the unequal heating of the gas in the discharge-tube, I turned the tube into a horizontal position, and then found that for air (the gas I was using at the time) the discharge was always bent vertically upwards, the deflexion being very large for high pressures of the gas, and vanishing for low pressure.

When the discharge passed, it could be seen to go, at first, straight between the electrodes, and then to be quickly carried upwards as the gas got heated, until a steady position 
was attained where the discharge remained permanently deflected ; for very high pressures of the gas in the dischargetube there was no steady position (see fig. 18, below); in this case the path of the discharge, after being bent upwards, always fell back to the straight line again, as if it were being blown out.

Fig. 6.

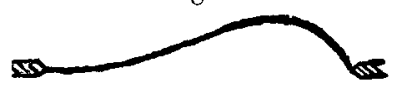

The curvature of the discharge, as deflected by convectioncurrents, was not nearly uniform for pressures in the dischargetube of 4 or 5 cms. of nercury, but appeared as shown in fig. 6 , the curvature being much greater near the negative electrode; as the pressure of the gas was diminished, the curve became more symmetrical until it finally became quite circular.

The discharge in carbonic acid gas was bent by convectioncurrents rather more than in air; but in hydrogen the effect was so small as to be almost imperceptible.

\section{Rearrangement of Apparatus.}

To get rid of the effect of the convection-currents in the photographs of the discharge, the direction of the magnetic force was made vertical, and that of the discharge horizontal, so that the horizontal component of the deflexion was due entirely to the magnetic force.

The camera had now to be fixed in a position vertically over the discharge, and with the sensitive plate horizontal. By this means the horizontal component only of the deflexion showed in the photographs, and the deflected discharge then appeared of nearly uniform curvature except for high pres. sures of the gas -3 to $4 \mathrm{cms}$. of mercury-when the curvature increased greatly towards the middle of the discharge; but with the point of maximum curvature always nearer to the negative electrode, as shown in fig. 7 , where $a$ is the negative electrode and $b$ the positive. On the other hand, for low pressures of the gas-less than a centim. of mercury-the curvature increased towards the positive end of the discharge. For a pressure of 1 millim. the discharge in air or carbonic acid gas was turned at right angles to the positive electrode by a strong magnetic force, and the positive column was deflected till it pressed against the sides of the tube.

Fig. 7.

In hydrogen the deflexion showed similar changes for low pressures, but on a smaller scale; for high pressures the 
deflexion was so small that irregularities in it could not be detected very well.

\section{Experiments.}

Experiments were next made to find how the deflexion of the discharge by magnetic force changed with-

(1) The pressure of the gas in the discharge-tube;

(2) The mean difference of potential between the electrodes of the discharge;

(3) The mean current in the discharge.

The method adopted in these experiments was to find the deflexions produced in the same gas by a constant magnetio force, first for different pressures of the gas while the mean current in the discharge was kept constant; and, second, for different strengths of current in the discharge, as measured. by the galvanometer, while the pressure of the gas was kept constant. The mean current carried by the discharge through the gas was controlled by a variable water-resistance, connected in series with the discharge-tube. Thus by increasing the water-resistance in circuit when the pressure of the gas in the discharge-tube fell, the mean current tbrough it was kept constant.

As the difference of potential between the electrodes depended for any gas on its pressure in the discharge-tube, so long as the distance between the electrodes remained unchanged, it could not be varied independently of the pressure; but, as we shall see, this did not matter.

\section{Details of Experiments.}

The length of the discharge in these experiments was $8.8 \mathrm{cms}$., and the gases most nsed were air and carbonic acid gas, especially the latter, owing to the large deflexions produced in it. In these gases when the pressure was less than $2 \mathrm{cms}$. of mercury, the discharge had a "fuzzy" appearance; and for pressures less than $1 \mathrm{~cm}$. the photographs obtained were too indefinite to be of any use. The highest pressure for which the discharge would pass properly between the electrodes was about $5 \cdot 5 \mathrm{cms}$.

\section{Results.}

The following figures are taken from photographs of the deflected discharge obtained during these experiments.

Figs. 8 and 9 show the effect of change of pressure in air and carbonic acid gas respectively, while the mean current in the discharge, as measured by the galvanometer, was kept 
Force of the Electric Discharge through Gases. 435 constant; figs. 10 and 11 show the effect of change of current in the discharge while the pressure of the gas was kept constant.

Fig. 8.

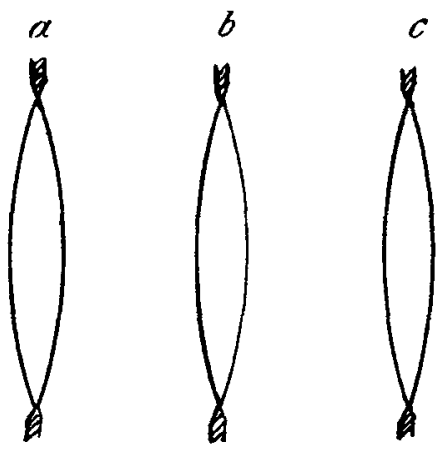

Fig. 9.

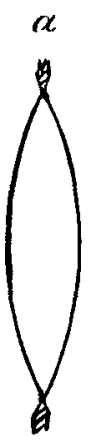

Fig. 10.
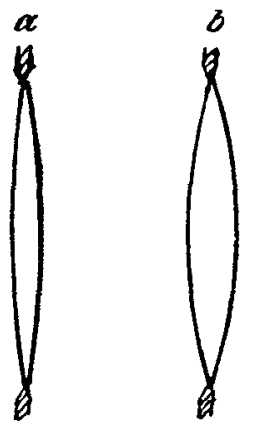
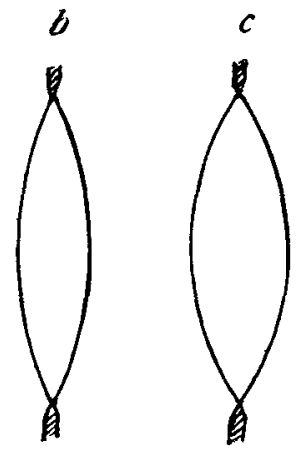

Fig. 11.

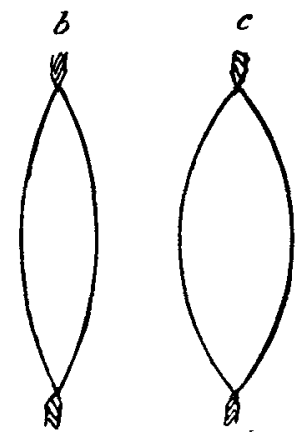


The following table gives for each figure the pressure of the gas in the discharge-tube in cms. of mercury, the

\begin{tabular}{|c|c|c|c|c|}
\hline Figures. & Gas. & $\begin{array}{c}\text { Mean } \\
\text { current. }\end{array}$ & $\begin{array}{c}\text { Pressure } \\
\text { of gas. }\end{array}$ & $\begin{array}{l}\text { Potential- } \\
\text { difference. }\end{array}$ \\
\hline $\begin{array}{r}8, a \ldots \ldots \\
8, b \ldots \ldots \\
8, c \ldots \ldots \\
9, a \ldots \ldots \\
9, b \ldots \ldots \\
9, c \ldots . . \\
9, d \ldots \ldots \\
10, a \ldots \ldots \\
10, b \ldots \ldots \\
10, c \ldots \ldots \\
11, a \ldots \ldots \\
11, b \ldots \ldots \\
11, c \ldots \ldots\end{array}$ & $\begin{array}{c}\text { Air } \\
" \\
\ddot{C O}_{2} \\
" \\
" \\
\text { Air } \\
" \ddot{O O}_{2} \\
" \\
"\end{array}$ & $\begin{array}{r}80 \\
80 \\
80 \\
95 \\
91 \\
155 \\
152 \\
44 \\
80 \\
275 \\
35 \\
215 \\
430\end{array}$ & $\begin{array}{l}1 \cdot 4 \\
2 \cdot 2 \\
3 \cdot 7 \\
3 \cdot 5 \\
0 \cdot 8 \\
4 \cdot 6 \\
2 \cdot 8 \\
3 \cdot 3 \\
1 \cdot 4 \\
1 \cdot 4 \\
4 \cdot 1 \\
4 \cdot 1 \\
1 \cdot 6\end{array}$ & $\begin{array}{r}1100 \\
1150 \\
1425 \\
1250 \\
965 \\
1490 \\
1320 \\
1225 \\
1100 \\
1120 \\
1475 \\
1495 \\
950\end{array}$ \\
\hline
\end{tabular}

mean current in the discharge in divisions of the galranometer-scale-one division of the scale corresponding to a current of $\frac{1}{120,000}$ of an ampere,-and the mean potential-difference between the electrodes in volts.

From these figures we see that there is very little, if any, change of deflexion with pressure so long as the current in the discharge is kept constant, but that the deflexion increases with the current whether the pressure of the gas is kept constant or not; the increase in the deflexion, as measured by the increased curvature of the path of the discharge, is not, however, proportional to the increase of the mean current in the discharge. Figs. 8 and 9 also show that the deflexion is independent of the potential-difference between the electrodes of the discharge.

The magnetic force deflecting the discharge in these experiments was nearly one C.G.S. unit, and the frequency of discharge was about 60 per sec.

\section{Tension in the Gas carrying a Discharge.}

The part played by the gas in modifying the deflexion of the discharge through it could be readily explained by supposing that the dissociated molecules or ions in the gas along the line of discharge are polarized and held in line by a sort of tension, this tension being different for different gases; on this assumption, the greater the tension in any gas the less would be the deflexion.

Assuming the path of the discharge to be held in position in this manner, and to be deflected like an elastic string by a uniform force everywhere perpendicular to the element of 
the string on which it acts, we have the following formula connecting the tension $\mathrm{T}$ along the discharge with $r$, the radius of curvature of the deflected path,

$$
\mathrm{T}=\mathrm{F} \cdot r \text {, }
$$

where $\mathrm{F}=$ the force deflecting the path of the discharge at any point

$$
=(\mathrm{c} \cdot t)
$$

where $\mathrm{C}$ is the current in the discharge, and $f$ is the magnetic foree at right angles to the discharge.

If $\mathrm{C}, r$, and $f$ are expressed in C.G.S. units, $\mathrm{T}$ in the above formula will be given in dynes.

On calculating $\mathrm{T}$, however, for different values of $\mathrm{C}$ and $r$ obtained by experiment, the values were found to differ widely for the same gas.

\section{The Nuture of the Deflexion by Magnetic Force.}

From the behaviour of the discharge when the magnetic field was suddenly made, or being made was quickly reversed in direction, it seemed more probable that the deflexion of the discharge by magnetic force was due to the same cause as that produced by the convection-currents in the gas, and that in either case the path of the discharge was determined by the line of least resistance through the gas between the electrodes. The discharge could hardly be deflected in the form shown in figs. 6,7 , or 18 if it were held in position by a tension along. the line of discharge.

It is well known that the electromotive force required to maintain a discharge in a rarefied gas is not nearly so great as that required to start it, since the molecules of the gas dissociated or ionized by the first discharge leave, as it were, a thread of conducting material between the electrodes, along which succeeding discharges pass much more easily than through fresh gas. When this thread of ionized gas is displaced by any means-e. g. magnetic force acting on the charged and moving particles of the gas, or by currents in the gas itself due to any cause and blowing across the electrodesthe discharge follows it, since the increase of the resistance due to the increase in length of the displaced path is more than compensated for by the greater conductivity in this direction.

If, however, the time between successive discharges is so great that the thread of ionized gas produced by one discharge becomes dissipated, or is destroyed in any way, before the next discharge takes place, then we should expect no deflexion 
from any cause unless each discharge lasted a sensible time ('Recent Researches,' p. 105, par. 90).

Support is given to this view by the fact observed throughout the experiments, that when the discharge was vertical a horizontal magnetic force always increased the potential-difference between the electrodes of the discharge, this increase being greatest when the deflexion of the discharge was greatestthe mean potential-difference was increased in some cases from 1000 to 1100 volts, - while in hydrogen, where the deflexion was hardly visible, the same magnetic force produced no perceptible increase in the potential-difference, as measured by the electrostatic voltmeter used. When the discharge was horizontal and at right angles to the magnetic force, the potential-difference between the electrodes was increased or diminished according as the magnetic force increased or diminished the deflexion of the discharge due to the convectioncurrents in the gas. In the latter case the magnetic force shortened the length of the discharge, and at the same time tended to keep the ionized gas together, thus reducing the resistance, while it had the opposite effect in the former case.

When the time between successive discharges was increased by using a hand-break for the primary current in the inductioncoil, the magnetic deflexion was diminished until, when the discharge was separated by nearly a second or more, each discharge began almost in the straight line between the electrodes, and was broadened as shown in fig. 12, the ionized gas being here deflected through the breadth of the band while the discharge lasted.

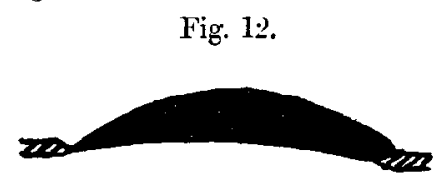

To get a visible broadening the pressure of the gas in the discharge-tube had to be low enongh to let the dischirge last some time: for high pressures of the gas the discharge passed almost straight across.

\section{Further Experiments.}

To try if the current in the discharge as measured by the galvanometer was proportional to the maximum current during the discharge, a magnetizing-spiral was connected in series with the discharge-tube; a bundle of steel wire carried in the end of a glass tube could be slipped into this spiral and 
then taken out and tested for its magnetism hy an ordinary magnetometer with lamp-and-scale arrangement.

From these experiments it was found that the maximum current in the discharge, as measured by its magnetizing effect on the bundle of wire in the spiral, was not at all proportional to the average current measured by the galvanometer. The experiments showed that while the maximum current increased with the pressure of the gas in the dischargetube, the average current, and with it the magnetic deflexion, decreased. Different kinds of wire, from soft iron to hard steel, were used for testing the maximum current; but all showed the same result.

In order to increase considerably the maximum current in the discharge without changing the pressure of the gan, leyden-jars were connected across the electrodes as shown in fig. 13 .

Fig. 13.

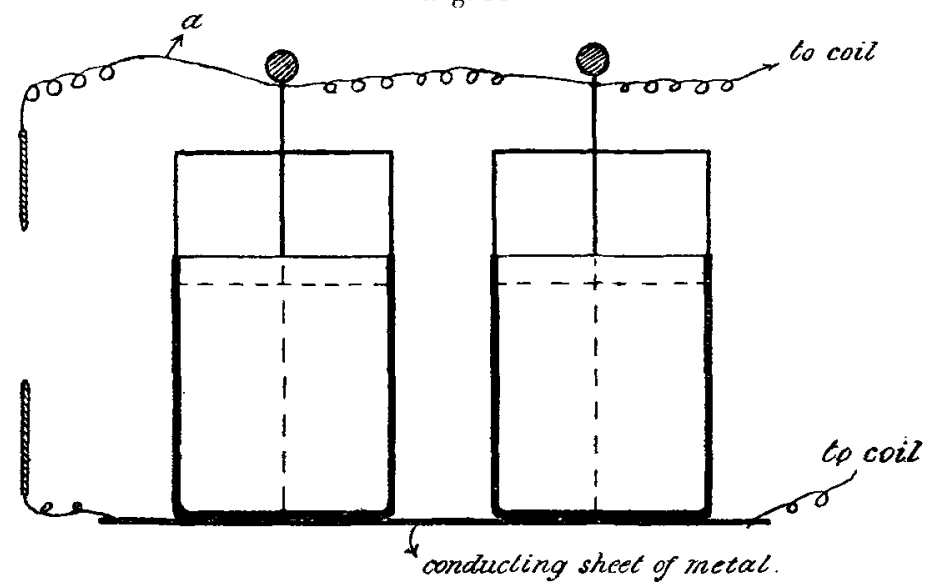

The inside coatings of the jars were connected together and to one terminal of the discharge-tube and the outer coatings to the other terminal.

The effect of this arrangement was to increase very much the intensity of the discharge and its manetizing power, but always to decrease the deflexion, whether caused by magnetic force or by convection-currents : this effect was increased by making a spark-gap in the discharge-cirenit at $\alpha$ in the diagram (fig. 13).

On examining this discharge in a rotating mirror $I$ found that it was, so far as the mirror showed, instantaneous; and as the duration of the discharge, from these and previous experiments, seemed to be very closely connected with its 
deflexion by magnetic force, experiments were next made to see how far this connexion went.

\section{Details of Experiments.}

In these experiments the discbarge-tube was placed between the poles of a powerful electromagnet so that a very intense magnetic field could be employed; the discharge-circuit was arranged in four different ways, and for each the duration and the deflexion of the discharge were observed, with the gas in the discharge-tube at different pressures.

(1) The discharge-tube was connected directly with the terminals of the secondary of the induction-coil.

(2) The same as (1), except that a spark-gap was made in one of the wires leading to the induction-coil.

(3) The arrangement shown in fig. 13.

(4) The same as (3), but with a spark-gap made in the circuit at the point marked $a$ in the diagram (fig. 13).

The duration of the discharge in each case was roughly estimated by the extent of the broadening of the image in a rotating mirror, and the magnetic deflexion by direct observation.

\section{Results obtained.}

In case (1) the deflexion produced by the magnetic force was small for high pressures of the gas in the discharge-tube, but increased greatly with a considerable broadening of the discharge as the pressure of the gas fell.

The duration of the discharge also increased as the pressure of the gas fell, and the broadening of the discharge, seen in the rotating mirror, seemed proportional to that produced by the magnetic force. For high pressures of the gas the discharge seemed instantaneous.

(2) The external spark-gap always diminished the deflexion greatly, but did not entirely destroy it, except for very high pressures of the gas in the discharge-tube. The effect of the spark-gap was greater the greater its length.

The appearance of this discharge in the rotating mirror was a bright line followed by a fainter band of varying breadth, depending inversely on the length of the spark-gap and the pressure of the gas in the discharge-tube. This fainter portion of the discharge was always more deflected than the bright part, and the latter was not sensibly broadened either by the maginet or by the mirror.

(3) Here the discharge for high pressures of the gas was practically instantaneous; no broadening could be seen in the rotating mirror, and the magnetic deflexion vanished. The 
discharge in this case was intensely bright, and gave the usual candle-spectrum of the gas in the discharge-tube.

As the gas was pumped out of the tube the appearance of the discharge changed gradually from being very bright, white, and sharply defined, to being dull, reddish coloured for air, and "fuzzy," its deflexion by magnetic force and also its duration increasing at the same time.

This discharge was separited both by the magnet and by the rotating mirror into two parts, the first being very bright, least deflected by the magnet, and lasting a very short time; the next not so bright, reddish coloured for air, deflected and broadened by the magnet, and of much longer duration than the first.

The discharge as deflected by magnetic force is sho.vn in fig. 14; the heavy line represents the bright part of the discharge, the hatched portion of the figure the fainter but

Fig. 14.

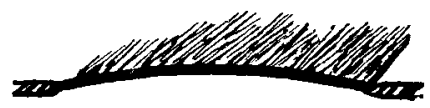

more deflected and broadened part. The same figure would represent the undeflected discharge as seen in the rotating mirror if the heavy line were straight instead of curved.

As the pressure of the gas in the discharge-tube was lowered still further the discharge became uniform in appearunce, both in the mirror and in the magnetic field, while the deflexion as well as the broadening and the duration of the discharge increased.

(4) In this case no deflexion could be observed at any pressure when the spark-gap was of sufficient length to ensure the whole discharge passing at once; for very low pressures of the gas in the discharge-tube the capacity of the jars had to be increased, as changing the length of the spark-gap alone was not sufficient to prevent the discharge trailing out.

In any of the above experiments a discharge that could be sensibly broadened in the rotating mirror was always deflected by the magnetic force.

The frequency of the discharge was about fifteen per second.

In an experiment arranged as in case (3) above the pressure of the gas was gradually reduced, until the coil was just able to send a discharge that was drawn out a little in the rotating mirror, and that had a slightly "fuzzy" appearance; but when the discharge passed for a little time it suddenly changed into a bright sharp discharge, nat visibly broadened in the rotating 
mirror. The magnetic force deflected this discharge at first, but immediately it changed its appearance it fell back to the straight line between the electrodes. The change in the character of the discharge was evidently due to the increase of pressure in the discharge-tube caused by the heating of part of the gas, as letting more gas into the tube would have produced the same changes. This experiment was repeated several times, but always with the same result : after stopping the discharge for a few seconds, it always started again as at first.

In hydrogen the deflexion is very small, but as in other gases it increases, though slowly, as the pressure of the gas falls. For the lowest pressure obtained-less than one millim. of mercury - the deflexion was very much greater than for pressures of one or two centims., yet it was not nearly so great as that obtained in air or carbonic acid gas for the same pressure.

The discharge in hydrogen, when examined in the rotating mirror, showed no appreciable broadening until the pressure of the gas in the discharge-tube was reduced sufficiently to allow a decided magnetic deflexion, and as the pressure was lowered still furth $\mathrm{r}$, the broadening of the discharge in the mirror, as well as the maguetic deflexion, continued to increase.

\section{Explanation of Results.}

These experiments point to the conclusion that the influence of the gas on the deflexion of the discharge in it is indirect. In any of the gases used, when precautions were taken to make the discharge abrupt, and to prevent it trailing out at the end, no deflexion could be observed, even in a very strong magnetic field produced by a large electromagnet with conical pole-pieces, the discharge-tube used in this case being small-one inch in diameter-to allow the pole-pieces to be brought near together so as to increase the magnetic force between them. But when the discharge was allowed to pass in the ordinary way, the deflexion always increased as the discharge became less abrupt and more prolonged as the pressure of the gas in the discharge-tube fell.

A very important factor in determining the magnitude of the deflexion in any gas for a given frequency of discharge, was its duration, the quantity of electricity carried by the discharge being of little importance.

This was shown by a comparison of the deflexions obtained in the experiments, case (4) above ; when the pressure of the gas in the discharge-tube was high, and a small leyden-jar was nsed instead of the two shown in fig. 13, the quantity of 
electricity carried by each discharge was very small, even when the pressure of the gas was low enough to allow the discharge to be appreciably deflected; but when the pressure of the gas was low and several large jars were used instead of the one small one, the quantity of electricity carried by each discharge through the gas was very much greater than in the former case, as was shown by the galvanometer, still the discharge was not visibly deflected; while the discharge carrying much less electricity in the former case, but lasting a longer time, as was shown by the rotating mirror, was deflected. When jars were used withont any spark-gap, as in case (3) above, the first part of the discharge, which carried the charge of the jars connected across the electrodes, was always least deflected (see fig. 14), while the less intense but more prolonged parts were always more deflected.

As the duration of the discharge in any gas depends on the pressure of the gas, the conclusion might seem to be at rariance with the results of previous experiments, where it was shown that the deflexion was independent of the pressure of the gas, provided the current in the discharge was kept constant; but in those experiments, to keep the current in the discharge constant when the pressure of the gas was lowered, a very large resistance had to be introduced into the discharge circuit, and this added resistance had the same effect in shortening the time of discharge as the reduction of the pressure had in increasing it: that adding resistance to the circuit had the effect of shortening the time of discharge was shown by the rotating mirror. In the experiments referred to, an increase of current in the discharge was always accompanied by an increase in its duration.

Influence of the Gas on the Deflexion of the Discharge.

With the explanation of the deflexion of the discharge by a magnetic force given by Professor Thomson (see p. 437 abore) the deflexion of the discharge should be influenced by the gas through which it passes in several ways:-

\section{(1) The Rate of Diffusion of the Gus.}

During the interval between successive discharges the dissociated or ionized gas left by one discharge scatters rapidly through the tube owing to diffusion, so that the conductivity along this path for the next discharge rapidly falls ; and if the diffusion is very rapid, the path of least resistance for each discharge may be the shortest one, i.e. the straight line between the electrodes, as in the case when the frequency of the discharge was made very small. 
(2) The Rate of Recombination of the Ionized Gas.

The recombination of the gas ionized by a discharge has much the same effect as diffusion in destroying the conductivity of the gas between successive discharges, rapid recombination acting like rapid diffusion.

(3) The Velocity of the Ions in the Gas.

The velocity of the ions in the gas under an electromotive force has an effect similar to (1) and (2), since the ions will be pulled towards the electrodes between successive discharges, thus destroying the conductivity of the path through the gas ; the greater the velocity of the ions the less would be the conductivity of the ionized gas left by a discharge for the following one.

For high pressures of the gas in the discharge-tube the effect of the velocity of the ions should be more marked, when an induction-coil is used to send the discharge. In this case the coil will only send a discharge for a break of the primary, while for the make there is an electromotive force acting on the gas between the electrodes pulling in the ions, though no discharge passes to renew the supply.

\section{(4) The Duration of the Discharge.}

The time given for causes (1), (2), and (3) to destroy the conductivity of the gas for a given frequency of discharge, is diminished by the length of time each discharge lasts, so that the greater the duration of each discharge the less will be the effect of (1), (2), and (3) in destroying the conductivity of the gas between successive discharges. For very low pressures of the gas in the discharge-tube, the time each discharge lasts, as shown by a rotating mirror, is a big part of the interval between two discharges produced by an induction-coil.

(5) The Conductivity of the Gas after a Discharge has passed through it, relative to that of the same Gas before the Discharge.

If a gas suffers little change from the passage of a discharge through it, and if its resistance to the first discharge is little greater than it is to succeeding discharges, then the path of the discharge should never be much deflected from the straight line between the electrodes, as the length of the path of least resistance for the discharge through the gas cannot in this case be much increased.

In hydrogen, for example, the potential-difference required to maintain a discharge between a pair of electrodes is much 
greater than that required in nitrogen or carbon monoxide (Recent Researches, p. 152, par. 150), although the potentialdifference necessary to begin the discharge is much less in hydrogen than in these gases. This effect, in hydrogen, may, however, be due to the very rapid scattering of the ionized gas produced by a discharge, from causes (1), (2) and (3) mentioned above.

The less a gas is ionized by a discharge through it, the less should its resistance be diminished for succeeding discharges, and the less should the discharge in it be capable of being deflected.

The resistance of the gas in the discharge-tube to the discharge passing through it will affect its duration, since the coil will continue to send the discharge until the potentialdifference between its terminals has fallen so much that it is not able any longer to force the current over the opposing resistance.

\section{The Discharge in Mercury Vapour.}

As it seemed probable that a monatomic gas would be less broken up, and would consequently have its resistance less changed, by the passage of a discharge through it than other gases, an experiment, was made to try the effect of magnetic force in deflecting the discharge in mercury vaponr.

To procure a good mercury vacuum, a tube of the form shown in fig. 15 (see p. 446) was employed. 'The vacuum-tube was partly filled with clean mercury $m$, and connected by the tube $d$ to a mercury air-pump with the capillary tube $c$ in a vertical position; the end of $c$ dipped under mercury in the test-tube $e$ as shown ; $e$ was more than 76 centims. long.

The vacuum was made by pumping until no more air conld be got out, then the mercury in the tube was heaied and slowly distilled for about two hours, the air-pump being worked at the same time.

While the mereury was being distilled, the discharge was kept going by an induction-coil and the platinum electrodes $a, b$ were raised several times to a white heat, to drive out any gas occluded in them. 'The vacuum-tube was then sealed off the air-pump at the constriction $d$, left for the purpose.

After distilling the mercury left in the bulb for about three hours more, so as to get rid of any traces of air left in it, the bulb was then placed between the poles of the electromagnet used in previous experiments, with the direction of discharge horizontal and perpendicular to the magnetic force. The mercury was then heated, and the effect of the magnet on the

Phil. Mag. S. 5. Vol. 46. No. 282. Nov. 18!8. 2 I 


\section{6}

Mr. J. Henry on the Deflexion by Magnetic

discharge between the electrodes, sent by an induction-coil, noted. The pressure of the mercury vapour in the bulb was first raised as bigh as possible by heating, and then allowed to fall by cooling the bulb, but in no case was the dischargo very much deflected. The discharge was bent upwards a little by the vertical current of vapour.

The experiment showed that the deflexion of the discharge in mercury vapour was very much less than in air or carbonic acid gas for low pressures, and seemed even less than in hydrogen.

Fig. 15.
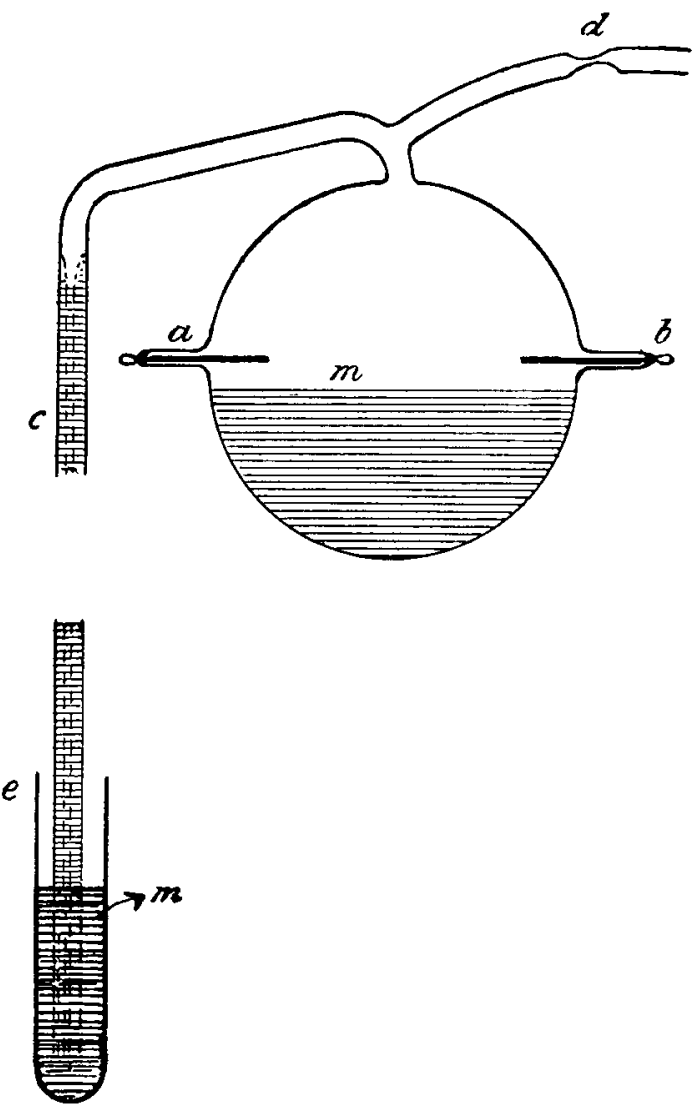

Comparison of Theory with Experiment.

Taking the gases-hydrogen, air, and carbonic acid gaswith which careful experiments were made, we find the mag- 
netic deflexion of the discharge in hydrogen very small, in air many times larger, but less than in carbonic acid gas.

This is what the explanation given above would lead one to expect, since all the causes mentioned above as tending to prevent deflexion are, so far as we know them, greatest for hydrogen and least for carbonic acid gas.

In this connexion I may mention that Mcclelland finds the velocity of the ions produced by an electric discharge many times-five or six-greater in air than in carbonic acid gas, and though he has not yet made a determination for hydrogen, there is no doubt the velocity of the ions in it is much greater than in any otber gas. $C f$. the velocities found by Rutherford for the ions produced by Röntgen rays (Phil. Mag. vol. xliv. p. 434, 1897).

\section{Experiments on the Frequency of Discliarge.}

In most of the above experiments, in order to make the discharge as steady and regular as possible, a tuning-fork mercury interrupter was employed for breaking the current in the primary of the induction-coil, instead of the ordinary vibrating contact interrupter belonging to the coil.

Some experiments were made to find whether, by increasing the pitch of the fork used for interrupting the current in the primary coil circuit, the frequency of the discharge could not be increased sufficiently to enable even the abrupt, short discharges to be deflected. These experiments, however, were not a success, as the induction-coil would not work with a fork making more than about 120 complete vibrations per second, and for a fork of this pitch the action of the coil was very weak, even when a dangerously high electromotive force was used in the primary circuit. The vibration frequency of the interrupter belonging to the coil was about 60 per second.

In these abrupt discharges the time between successive discharges is practically the same as the time of vibration of the interrupter in the primary circuit of the induction-coil, since each discharge is almost instantaneous, whereas in the ordinary discharge the current begins gradually in the discharge-tube, rises to a maximum, and falls away gradually again, in accord with the rise and fall of electromotive force in the secondary of the coil. In this way the interval between successive discharges during which the ionized path through the gas may be destroyed is diminished, and when the resistance in the discharge circuit is small-when the pressure of the gas in the discharge-tube is low-this interval may become small compared with the time of vibration of the interrupter in the primary-coil circuit. 
That this is the case is shown pretty clearly by Feddersen's observations (Pogg. Ann. ciii. p. 69, 1858) on the appearance presented by a succession of sparks in a revolving mirror, when a blast of air was directed across the electrodes (Recent Researches, p. 132, par. 128).

Feddersen's sparks were obtained from the oscillatory discharge of leyden-jars, and the blast was simply that caused by the rotation of the mirror. Each discharge gave a series of sparks following one another very rapidly, the sparks at the beginning of each series being less separated in the revolving mirror than those towards the end of the series.

In these experiments the first spark in each discharge was observed to pass straight between the electrodes, while the succeeding ones were more and more bent by the blast, as shown in fig. 16 : this figure gives the appearance presented by the sparks at different parts in the series of oscillations forming one discharge; $a$ is the beginning of the series, and $b$ the end.

a

Fic. 16.
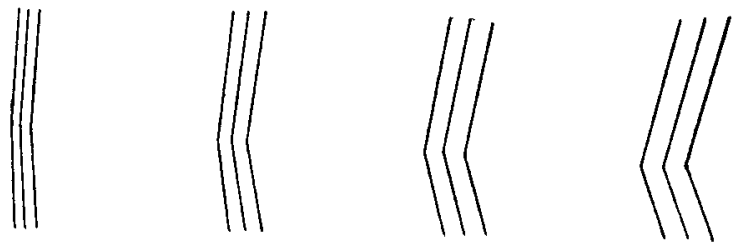

These observations show that between successive discharges the ionized gas was blown away or scattered, so that each discharge found it easier to begin along the straight path: but the interval between successive sparks in the same discharge was so small that the thread of ionized gas was bent only a little more by the blast each time while it retained its cunductivity; the increase in the deflexion between successive sparks, especially at the beginning of a series, was very small, but before the end of a series was reached the bending was very evident, as at $b$, fig. 16 .

When the electrodes of the secondary of an induction-coil are placed near enough together to form an arc, the gentle current of air across the electrodes produced by the heat of the discharge bends the are upwards; if the current of air be much increased, instead of bending the arc still more it will destroy the are altogether, and the discharge will pass as a spark straight between the electrodes with an aureole blown aside. A jlast of air is used to destroy in this way the sparking at the commutators in some dynamos. 
The spark-discharge of the induction-coil is not visibly bent by a blast. The blowing-out of the discharge was frequently observed during the experiments on the deflexion by magnetic force (p. 433 above).

An interesting case, showing the similarity between the action of magnetic force and of convection-currents in the gas, in deflecting the discharge, is shown in figs. 17 and 18 .

Fig. 17.

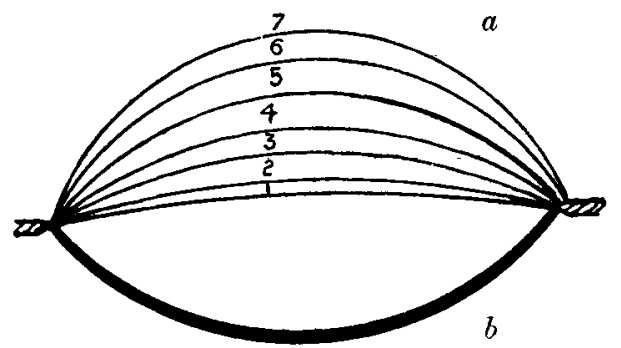

The large discharge-tube was filled with air, and exhausted until the coil was just able to send the discharge - the direction of the discharge was horizontal - when it was found that instead of remaining permanently deflected, the path of the discharge rose and fell; the first discharge was nearly straight, the second was more bent, and so on for four or five discharges, as shown in fig. 18, the discharge then fell to the straight line as if it had been blown out, and was blown aside again, the convection-currents in the gas being too strong to allow the discharge to preserve a permanent path.

Fig. 18.

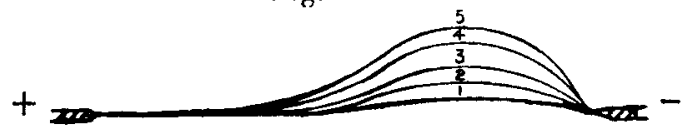

In fig. 17 the pressure of the gas was reduced until the discharge passed freely, and maintained a permanent path deflected by convection-currents, as $b$, fig. 17, only turned upwards, the convection-currents being always vertical.

Now when the magnetic field was made so as to bend the discharge upwards still more, it presented the appearance shown in fig. 17,a. The discharge rises and falls as before, the increase in the blast across the electrodes due to the magnetic field being sufficient to blow out the ionized path. When the magnetic force deflected the discharge downwards against the convection-currents, the discharge passed steadily, as shown at $b$, fig. 17 . 
Experiments in Blowing the Discharge in a Rarefied Gas.

Experiments were made to see if the discharge in hydrogen, at pressures less than atmospheric, could not be blown aside, as it was in other gases by the convection-currents in them due to the heat of the discharge.

For this purpose the tube shown in fig. 19 was employed.

Fig. 10.

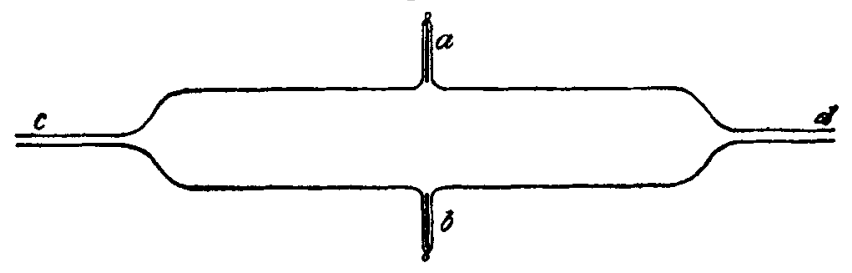

The discharge passed across the tube between the aluminium electrodes $a$ and $b$; the diameter of the tube was $2 \cdot 5$ centim., and the ends of the tube were drawn out, so that rubber pressure-tubing could be slipped on them ; in this way the end $c$ of the tube was connected through a stop-cock $k$ (not shown in fig.) to a large vessel A of 2045 cub. centim. capacity, and the end $d$ to a large ressel $\mathrm{B}$, and to the air-pump and manometer.

To begin an experiment the tube and vessels connected with it were filled with the gas to be experimented with, then the stop-cock $k$ was closed and the gas pumped out of the tube and vessel $B$.

A current of gas could now be blown across the electrodes by opening the stop-cock $k$, the strength of the blast depending on the difference of pressures between the gas in A and that in $\mathrm{B}$.

In this way the discharge in air or carbonic acid gas could be blown aside very readily when the pressure of the gas in the tube was not high enough to make the discharge pass as a bright sharp spark. By partially exhausting $A$, and then further exhausting $\mathrm{B}$, the mean pressure of the gas in the discharge-tube while the blast through it lasted could be varied at pleasure.

When the gas used was hydrogen, the blast across the electrodes when the stop-cock $k$ was opened bent the discharge very slightly for any pressure of the gas in the dischargetube. A strong blast had less effect than a rather gentle one : $c f$. the blowing out of an arc mentioned above.

By filling the vessel $\mathrm{A}$ and the tube with gas, vessel B being cut off, and then steadily pumping, the stop-cock $k$ 
being open, while the discharge passed between the electrodes, the effect of the current of gas across the electrodes produced in this way was observed for all pressures of the gas in the discharge-tube: of course the blast through the discharge diminished with the pressure of the gas and finally vanished.

When air or carbonic acid gas was used, the deflexion produced in this way was at first nothing, but gradually increased, attained a maximum, and then died away again as the vessel A became exhausted. In hydrogen the deflexion produced was always very small, and the maximum deflexion was produced after exhaustion had gone much further than in the case of air or carbonic acid gas, and consequently when the blast was weaker.

The velocity of the blast in air through the discharge-tube produced by this pumping method was about 2 centim. per second, when the pressure of the gas was about 14 centim. of mercury, and the discharge was bent as shown in fig. 21, though bardly so much.

The velocity of the blast in hydrogen, for about the same pressure of the gas, was 3 centim. per second, but the deflexion produced in the path of the discharge was hardly perceptible; blasts of smaller velocities for different pressures were also used, in addition to the strong blasts obtained by the first method, i.e. by exhausting vessel $\mathrm{B}$, and then opening the stop-cock $k$ until the pressures in $\mathrm{A}$ and $\mathrm{B}$ became equal by a flow of gas through the discharge-tube, but in no case was the discharge in hydrogen much deflected.

Fig. 20.

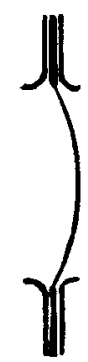

Fig. 21.

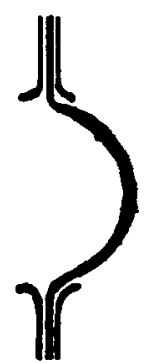

Fig. 20 shows the deflexion in hydrogen, about the maximum observed. Fig. 21 shows the deflexion in air, the actual deflexion was even greater in some cases.

When the blast through the discharge-tube was very strong, the deflexion in either air or hydrogen diminished. 
and the discharge became unsteady, as shown in figs. 17 and 18. The blast required to produce this effect for a given pressure of the gas was much less in hydrogen than in air.

From these experiments it is quite clear that the ionized path produced by a discharge is dissipated much more rapidly in hydrogen than in air or carbonic acid gas, otherwise the path of the discharge should be bent as the ionized gas is blown aside.

\section{Conclusion.}

The experiments described in this paper show that the deflexion of the electric discharge by magnetic force, and the deflexion of the discharge by a blast across the electrodes, are affected by the gas through which the discharge passes in exactly the same way; and that the properties of a gas on which the magnitude of the deflexion of the discharge in it depends are as stated at length on pages 443 and 444 :-

(1) The rate of diffusion of the gas.

(2) The rate of recombination of the gas ionized by a discharge.

(3) The velocity of the ions in the gas under an electromotive force.

(4) The duration of the discharge.

(5) The relative resistance of the gas to the passage of electricity through it before, and after, a discharge has passed.

Also for a given gas, the greater the frequency of the discharge the more readily is the discharge deflected, the deflexion vanishing for very slow rates of discharge.

For discharges following one another with very great rapidity we should still expect to get the magnitude of the deflexion produced by magnetic force, or by a blast, different in different gases unless (5) above is the same in all gases.

In conclusion I desire to acknowledge my indebtedness to Professor Thomson for the valuable assistance his snggestions have afforded me.

Cavendish Laboratory, 20 th June, 1898. 